Special Issue of the 6th International Congress \& Exhibition (APMAS2016), Maslak, Istanbul, Turkey, June 1-3, 2016

\title{
Characterization of 2D Hybrid Cellular Automata with Periodic Boundary
}

\author{
E. $\operatorname{ACAR}^{a, *}, \mathrm{~S} . \mathrm{UGUZ}^{a}$ AND H. AKIN ${ }^{b}$ \\ ${ }^{a}$ Department of Mathematics, Harran University, Sanliurfa, 63120, Turkey \\ ${ }^{b}$ Department of Mathematics, Zirve University, 27260, Gaziantep, Turkey
}

\begin{abstract}
We investigate main theoretical aspects of two-dimensional linear-hybrid cellular automata with periodic boundary condition over the Galois field GF(2). We focus on the characterization of two-dimensional hybrid linear cellular automata by way of a special algorithm. Here we set up a relation between reversibility of cellular automata and characterization of two-dimensional hybrid linear cellular automata with a special boundary conditions, i.e. periodic case. The determination of the characterization problem of special type of cellular automaton is studied by means of the matrix algebra theory. It is believed that this type of cellular automata could find many different applications in special case situations, e.g. image processing area, textile design, video processing, DNA research, etc., in the near future.
\end{abstract}

DOI: 10.12693/APhysPolA.131.432

PACS/topics: 02.10.Yn, 02.10.Ox, 07.05.Kf

\section{Introduction: $2 \mathrm{D}$ cellular automata}

Cellular automatons $(\mathrm{CA})$ are special discrete dynamic systems, the behavior of which is entirely characterized by some defined special, locally given rules. Up to now researchers have investigated and explored many aspects of CA with special interest in the study of different kinds of CA. These studies are mainly special analytic ideas into the behavior of the iterative process. Cellular automata were initially investigated by von Neumann and Ulam [1] around 1950's and were systematically investigated by Hedlund, considering just theoretical view. One-dimensional (1D) CA were studied mathematically very extensively and many various applications of CA were demonstrated. However, not so much interest is accorded to 2D uniform and hybrid CA. Von Neumann has stated that a CA can be more universal, considering its many elegant properties [1]. Due to their structural complexity, von Neumann rules have never proceeded into the computer program language codes at that time. Around the eighties, S. Wolfram [2] has started to study some prominent properties of simple $1 \mathrm{D}$ uniform CA rules and has found that even different simple rules are capable of showing very interesting complex and chaotic behavior. Some basic and unified mathematical CA models, considering properties of matrix linear algebra over the binary field $\mathrm{Z}_{2}$, that specify the behaviors of $2 \mathrm{D}$ linear nearest neighborhood of uniform CA with null or periodic boundary conditions, can be found in the literature $[3,4]$. Uniform linear CA has been accorded a striking interest in the past decades [4-19]. Due to its structure and elegant properties, CA has an opportunity to model many scientific computational problems, to understand many similar model behaviors and sometimes to easily explain

*corresponding author; e-mail: karakusecem@harran.edu.tr their nature. In $[4,20]$ the mathematical analytic behavior of the hybrid and uniform $2 \mathrm{D} \mathrm{CA}$ with states values in binary fields $\mathrm{Z}_{2}$ were investigated.

Some CA reports mainly investigate the characterization of the uniform $1 \mathrm{D}$ and $2 \mathrm{D}$ cellular automata. In this paper, we concentrate on a special family of $2 \mathrm{D}$ finite linear-hybrid cellular automata with periodic condition over the Galois field GF(2). In other words, it is studied a two-state CA problem for a specific transformation, with some fixed conditions. Here, we set up a specific relation between reversibility of hybrid cellular automata and characterization of $2 \mathrm{D}$ hybrid $\mathrm{CA}$ with periodic boundary conditions. We study the determination of the characterization problem of this special cellular automaton by means of the matrix algebra theory. Due to CA nature, it is very simple to allow some important mathematical studies and to obtain very complicated and complex behavior of chaos in dynamical systems. It is believed that hybrid linear CA can have many different kinds of applications. Using the linear rule matrices presented in the work, the presented results give further insight into the algebraic consequences of these 2D hybrid CA and relate to some elegant applications found by the authors in the literature (i.e. $[3,8-23]$ ).

\section{Preliminaries and rule matrices of uniform CA primary rules}

Considering the neighborhood of the information cells, there are two well-known studied boundary approaches in the literature.

- Null or fixed boundary: the boundary cells are zero spin values, or fixed 0 -state.

- Periodic boundary: the boundary cells are contiguous in each border direction. 
An important note is that, there are also different types of boundary conditions to study in future, i.e. reflexive, adiabatic etc. (see details in [15-19]).

$$
T_{1 N}=\left(\begin{array}{ccccccc}
0 & 1 & 0 & 0 & \cdots & 0 & 0 \\
0 & 0 & 1 & 0 & \cdots & 0 & 0 \\
0 & 0 & 0 & 1 & \cdots & 0 & 0 \\
0 & 0 & 0 & 0 & \cdots & 0 & 0 \\
\vdots & \vdots & \vdots & \vdots & \ddots & \vdots & \vdots \\
0 & 0 & \cdots & 0 & 0 & 0 & 1 \\
0 & 0 & \cdots & 0 & 0 & 0 & 0
\end{array}\right),
$$$$
\text { 2.1. Uniform } C A \text { rule matrices }
$$

Lemma 1 [3]. For uniform type of CA, the representation of the fundamental linear CA rule matrices $\left(2^{0}=1\right.$, $2^{1}=2,2^{2}=4,2^{3}=8,2^{4}=16,2^{5}=32,2^{6}=64$, $2^{7}=128$ and $2^{8}=256$ ) with null boundary neighborhood over $\mathrm{Z}_{2}$ is obtained considering the fixed $\mathrm{T}_{1 N}$ and $\mathrm{T}_{2 N}$ matrices presented above and is given as below:

Rule 1NB: $\left[X_{t+1}\right]=\left[X_{t}\right]$,

Rule 2NB: $\left[X_{t+1}\right]=\left[X_{t}\right]\left[T_{2 N}\right]$,

Rule 4NB: $\left[X_{t+1}\right]=\left[T_{1 N}\right]\left[X_{t}\right]\left[T_{2 N}\right]$,

Rule 8NB: $\left[X_{t+1}\right]=\left[T_{1 N}\right]\left[X_{t}\right]$,

Rule 16NB: $\left[X_{t+1}\right]=\left[T_{1 N}\right]\left[X_{t}\right]\left[T_{1 N}\right]$,

Rule 32NB: $\left[X_{t+1}\right]=\left[X_{t}\right]\left[T_{1 N}\right]$,

Rule 64NB: $\left[X_{t+1}\right]=\left[T_{2 N}\right]\left[X_{t}\right]\left[T_{1 N}\right]$,

Rule 128NB: $\left[X_{t+1}\right]=\left[T_{2 N}\right]\left[X_{t}\right]$,

Rule 256NB: $\left[X_{t+1}\right]=\left[T_{2 N}\right]\left[X_{t}\right]\left[T_{2 N}\right]$.

\subsection{Uniform $C A$ rule matrices under periodic boundary}

$$
T_{1 P}=\left(\begin{array}{ccccccc}
0 & 1 & 0 & 0 & \cdots & 0 & 0 \\
0 & 0 & 1 & 0 & \cdots & 0 & 0 \\
0 & 0 & 0 & 1 & \cdots & 0 & 0 \\
0 & 0 & 0 & 0 & \cdots & 0 & 0 \\
\vdots & \vdots & \vdots & \vdots & \ddots & \vdots & \vdots \\
0 & 0 & \cdots & 0 & 0 & 0 & 1 \\
1 & 0 & \cdots & 0 & 0 & 0 & 0
\end{array}\right),
$$

$$
T_{2 P}=\left(\begin{array}{ccccccc}
0 & 0 & 0 & 0 & \cdots & 0 & 1 \\
1 & 0 & 0 & 0 & \cdots & 0 & 0 \\
0 & 1 & 0 & 0 & \cdots & 0 & 0 \\
0 & 0 & 1 & 0 & \cdots & 0 & 0 \\
\vdots & \vdots & \vdots & \vdots & \ddots & \vdots & \vdots \\
0 & 0 & \cdots & 0 & 1 & 0 & 0 \\
0 & 0 & \cdots & 0 & 0 & 1 & 0
\end{array}\right)
$$

Lemma 2 [16]. For uniform type of $\mathrm{CA}$ over $\mathrm{Z}_{2}$, the representation of the fundamental linear $\mathrm{CA}$ rule matri$\operatorname{ces}\left(2^{0}=1,2^{1}=2,2^{2}=4,2^{3}=8,2^{4}=16,2^{5}=32\right.$, $2^{6}=64,2^{7}=128$ and $2^{8}=256$ ) with periodic boundary neighborhood over $\mathrm{Z}_{2}$ is found considering the fixed matrices $T_{1 P}$ and $T_{2 P}$ defined above and is given as follows:

Rule 1PB: $\left[X_{t+1}\right]=\left[X_{t}\right]$,

Rule 2PB: $\left[X_{t+1}\right]=\left[X_{t}\right]\left[T_{2 P}\right]$,

Rule 4PB: $\left[X_{t+1}\right]=\left[T_{1 P}\right]\left[X_{t}\right]\left[T_{2 P}\right]$,

Rule 8PB: $\left[X_{t+1}\right]=\left[T_{1 P}\right]\left[X_{t}\right]$,

Rule 16PB: $\left[X_{t+1}\right]=\left[T_{1 P}\right]\left[X_{t}\right]\left[T_{1 P}\right]$,

Rule 32PB: $\left[X_{t+1}\right]=\left[X_{t}\right]\left[T_{1 P}\right]$,

Rule 64PB: $\left[X_{t+1}\right]=\left[T_{2 P}\right]\left[X_{t}\right]\left[T_{1 P}\right]$,

Rule 128PB: $\left[X_{t+1}\right]=\left[T_{2 P}\right]\left[X_{t}\right]$,

Rule 256PB: $\left[X_{t+1}\right]=\left[T_{2 P}\right]\left[X_{t}\right]\left[T_{2 P}\right]$.

\section{Characterization and reversibility of hybrid linear CA (170PB-171PB)}

Considering the general case of the $\mathrm{CA}$ reversibility problem, the verification of reversibility of all 1D, 2D uniform or hybrid $\mathrm{CA}$ is a challenging problem. In other words, up to now there does not exist an algorithm for any general CA rule matrix situation. Here the significance of the reversibility phenomena of $2 \mathrm{D}$ linear $\mathrm{CA}$, or understanding of a general case, is shortly emphasized. The kernel dimension of the rule matrix of uniform or hybrid CA presents a hint or path to check the states of the iterative phase diagram and to detect CAs reversibility or non-reversibility. Hence, to obtain the kernel dimension of a 2D uniform or hybrid CA, the rule matrix rank values can be studied, i.e. the rank of $\left(T_{\text {rules }}\right)_{m n \times m n}$. Considering a finite situation of uniform or hybrid CA, many researchers have studied the corresponding CA rule matrices in order to find the inversibility of a $2 \mathrm{D}$ finitelinear-hybrid or uniform CA. Firstly the rule matrices $T_{\text {rules }}$, corresponding to finite $2 \mathrm{D}$ linear-hybrid CA, are found, then they are characterized considering the reversibility problem of hybrid CA. The following iteration property among the column vectors $X^{(t)}$ can be stated, with the rule matrix $T_{\text {rules }}: X^{(t+1)}=T_{\text {rules }} X^{(t)}(\bmod 2)$. If the transition rule matrix $T_{\text {rules }}$ is a non-singular matrix, then we have

$$
X^{(t)}=\left(T_{\text {rules }}\right)^{-1} X^{(t+1)}(\bmod 2) .
$$


Here, the main problem related to the $T_{\text {rules }}$ is whether the rule matrix $T_{\text {rules }}$ is an invertible matrix or not for any cases. Some know that any 2D finite CA is called a reversible CA if and only if CAs rule matrices $T_{\text {rules }}$ are non-singular (see [15-19] for extra details). If the transition matrices $T_{\text {rules }}$ of a $\mathrm{CA}$ have full rank properties, then it is said to be an invertible CA. Hence the 2D finite hybrid or uniform CA is a reversible one, otherwise it is called an irreversible CA. The analysis on reversible or irreversible cases of the transition matrices, located in the next subsection, is given by a specific theorem called Theorem (even). Thus, we study the use of the following structure, related to reversibility of CA in this section and detect the reversibility problem for $m \times n$, i.e $m$ is even or $m$ is odd cases.

In the present study, we work with special 2D CA defined by hybrid linear rules over the field $Z_{2}$ under periodic boundary case. We will determine the transition matrix $T_{\text {rules }}$. Firstly, a periodic boundary transition matrix rule $X$ of matrix of order $m \times n$ is considered. After that one can put 1 at $(1,1)$ position of this matrix and compute a specific selected linear rule number (170PB) for each of the $m n$ matrix elements, to obtain the first column of the transition matrix $T_{\text {rules. }}$. In addition the 1-elements of the first matrix $X$ are moved to the $(1,2)^{t h}$ element and the same procedure is applied in a repeated way, to obtain the second column of elements. The procedure is followed for all cell-elements of the first row elements of the first matrix $X$. Moreover, another linear rule (171PB) is applied for all cell-elements of the second row of the first matrix $X$. Using this procedure, the transition rules $170 \mathrm{~PB}$ and $171 \mathrm{~PB}$ will be applied.

As a finite example, let us take $m=3$ and $n=3$ and obtain the rule matrix $T_{\text {rule }}$ as below:

$$
T_{\text {hybrid }}=\left(\begin{array}{ccc}
S & I & I \\
I & S+I & I \\
I & I & S
\end{array}\right),
$$

where block matrix element $S, I$ are of $3 \times 3$ order:

$$
\begin{aligned}
& S=\left(\begin{array}{lll}
0 & 1 & 1 \\
1 & 0 & 1 \\
1 & 1 & 0
\end{array}\right), \\
& I=\left(\begin{array}{lll}
1 & 0 & 0 \\
0 & 1 & 0 \\
0 & 0 & 1
\end{array}\right) .
\end{aligned}
$$

For a general case the following is obtained. If $m$ is even, then matrix $T_{\text {rules }}$ is obtained as the following structure:

$$
T_{\text {hybrid }}=\left(\begin{array}{cccccc}
S & I & 0 & \ldots & 0 & I \\
I & S+I & I & \ldots & 0 & 0 \\
0 & I & S & \ldots & 0 & 0 \\
: & : & : & : & : & : \\
: & : & : & : & : & : \\
I & 0 & 0 & \ldots & I & S+I
\end{array}\right) .
$$

If $m$ is $o d d$, then $T_{\text {rules }}$ is constructed as given:

$$
T_{\text {hybrid }}=\left(\begin{array}{cccccc}
S & I & 0 & \ldots & 0 & I \\
I & S+I & I & \ldots & 0 & 0 \\
0 & I & S & \ldots & 0 & 0 \\
: & : & : & : & : & : \\
: & : & : & : & : & : \\
I & 0 & 0 & \ldots & I & S
\end{array}\right) .
$$

\subsection{Even case: the rank computations of $T_{\text {hybrid }}$ for $m$ even}

Consider $T_{i}$ indicating the $i^{\text {th }}$ row element and $T_{i}[j]$ indicating the $j^{t h}$ position element of the $i^{\text {th }}$ row of transition matrix $T_{\text {rules }}$, respectively. First, let us transfer the first column of the transition rule matrix in which $m$ is even to the last column, then the following is obtained:

$$
T_{\text {hybrid }}=\left(\begin{array}{cccccc}
I & 0 & 0 & \ldots & I & S \\
S+I & I & 0 & \ldots & 0 & I \\
I & S & I & \ldots & 0 & 0 \\
: & : & : & : & : & : \\
: & : & : & : & : & : \\
0 & 0 & 0 & \ldots & S+I & I
\end{array}\right) .
$$

Theorem (Even) 3: Let us consider the matrix

$$
\left(T_{\text {hybrid }}\right)_{m n \times m n}=\left(\begin{array}{cccccc}
I & 0 & 0 & \ldots & I & S \\
S+I & I & 0 & \ldots & 0 & I \\
I & S & I & \ldots & 0 & 0 \\
: & : & : & : & : & : \\
0 & 0 & 0 & \ldots & S+I & I
\end{array}\right),
$$

with $m, n \geq 3$, representing the two dimensional hybrid linear cellular automata over the field $Z_{2}$ under periodic boundary condition. Let $T_{1}^{1}=T_{1}, T_{1}^{k+1}=$ $-T_{1}^{k}[k](I)^{-1} T_{k+2}+T_{1}^{k}, T_{2}^{k+1}=-T_{2}^{k}[k](I)^{-1} T_{k+2}+T_{2}^{k}$ for $1 \leq k \leq m-2$. Let us denote $Q$ by $2 \times 2$ block matrix, consisting of square matrices of $n \times n$ :

$$
Q=\left(\begin{array}{ll}
T_{1}^{m-1}[m-1] & T_{1}^{m-1}[m] \\
T_{2}^{m-1}[m-1] & T_{2}^{m-1}[m]
\end{array}\right) .
$$

Then,

$$
\operatorname{rank}\left(T_{\text {hybrid }}\right)=(m-2) n+\operatorname{rank}(Q) .
$$

Proof: Using induction on $m$, we will find the linear algebraic relation of the rank properties of $T_{\text {hybrid }}$ for any case.

$$
T_{\text {hybrid }}=\left(\begin{array}{cccccc}
I & 0 & 0 & \ldots & I & S \\
S+I & I & 0 & \ldots & 0 & I \\
I & S & I & \ldots & 0 & 0 \\
0 & I & S+I & \ldots & 0 & 0 \\
: & : & : & : & : & : \\
0 & 0 & 0 & \ldots & I & 0 \\
0 & 0 & 0 & \ldots & S+I & I
\end{array}\right) .
$$

Firstly, one can see above that the sub-matrix consisting of all rows is the upper triangular form, except 
the first and second rows, and sub-matrix is a full rank matrix, such that the sub-matrix rank is $(m-2) n$. Then, one can multiply the third row element $T_{3}$ by $-T_{1}^{1}[1] I^{-1} T_{3}^{1}[1]$ and add that product to $T_{1}^{1}[1]$ element, so the first entry elements of the first new row $T_{1}^{2}$ will be zero. Also, the first row elements can be replaced by $T_{1}^{2}=-T_{1}^{1}[1] I^{-1} T_{3}+T_{1}^{1}$. Now we want $T_{1}^{2}[2]$ to be zero. In this case, we apply the same procedure to $T_{1}^{2}$. Thus, we have $T_{1}^{3}=-T_{1}^{2}[2] I^{-1} T_{4}+T_{1}^{2}$. Thus, the second entry-elements of the new first row become zero. Repeatedly, we see that after subsequent $m-1$ steps, the only non-zero entries of $T_{1}^{m-1}$ are $T_{1}^{m-1}[m-1]$ and $T_{1}^{m-1}[m]$. In similar way, by re-applying row calculations to the second row-elements, the only non-zero elements of the second row-entries are $T_{2}^{m-1}[m-1]$ and $T_{2}^{m-1}[m]$.

$$
\begin{gathered}
T_{\text {hybrid }}=\left(\begin{array}{cccccc}
0 & 0 & 0 & \ldots & T_{1}^{m-1}[m-1] & T_{1}^{m-1}[m] \\
0 & 0 & 0 & \ldots & T_{2}^{m-1}[m-1] & T_{2}^{m-1}[m] \\
I & S & I & \ldots & 0 & 0 \\
0 & I & S+I & \ldots & 0 & 0 \\
: & : & : & : & : & : \\
0 & 0 & 0 & \ldots & I & 0 \\
0 & 0 & 0 & \ldots & S+I & I
\end{array}\right) \\
=\left(\begin{array}{cc}
0 & Q \\
A & B
\end{array}\right) .
\end{gathered}
$$

The left lower block matrix of $A$ has full-rank, i.e. $\operatorname{rank}(A)=(m-2) n$. Hence we obtain that the rank equality of the transition matrix is presented by $\operatorname{rank}\left(T_{\text {hybrid }}\right)=(m-2) n+\operatorname{rank}(Q)$.

Example 4: Let us take an example of $m=3, n=3$. Then rule matrix is

$$
T_{\text {hybrid }}=\left(\begin{array}{ccc}
I & 0 & 0 \\
S+I & I & 0 \\
I & S & I
\end{array}\right) .
$$

Computation of the rank of $T_{\text {hybrid }}$ gives 9 . On the other hand, if someone apply Theorem 3 (even), then it is proceeded in the following way:

$$
\begin{aligned}
& T_{1}^{1}=T_{1}=[I, 0,0], \\
& T_{1}^{2}=-T_{1}^{1}[1] T_{3}+T_{1}^{1}= \\
& \quad I[I, S, I]+[I, 0,0]=[0,-S,-I], \\
& T_{2}^{2}=-T_{2}^{1}[1] T_{3}+T_{2}^{1}=-(S+I)[I, S, I] \\
& \quad+[S+I, I, 0]=\left[0,-S^{2}-S+I,-S-I\right] .
\end{aligned}
$$

Thus

$$
Q=\left(\begin{array}{ll}
T_{1}^{2}[2] & T_{1}^{1}[3] \\
T_{2}^{2}[2] & T_{2}^{2}[3]
\end{array}\right)=\left(\begin{array}{cc}
-S & -I \\
-S^{2}-S+I & -S-I
\end{array}\right) .
$$

Then $\operatorname{rank}(Q)=6$. Hence, $\operatorname{rank}\left(T_{\text {hybrid }}\right)=(m-2) n+$ $\operatorname{rank}(Q)=9$.
Remark 5: For the computation of $m$-odd case, similar approach is applied for the rank computations of $m$ odd $T_{\text {hybrid }}$ as given in Section 3.1. Due to the space shortage, we have skipped the details.

\section{Conclusions}

In this paper, the theoretical aspects of 2-dimensional hybrid-linear CA with periodic boundary case over twostates $Z_{2}$ are studied. A theorem for determining the reversibility of these CAs is presented for a general case of hybrid linear transformation. Other characteristics and different applications on 2D finite linear-hybrid CAs, by considering matrix algebra relations over three states $Z_{3}$, are investigated in the following studies. Additionally, after construction of the transition matrix representation of 2D hybrid-linear CA, one can find some algorithms to find the number of Garden of Eden configurations for the 2D hybrid-linear CA. It is another goal of this study. It is believed that CA hybrid theory could be applied successfully, especially in image processing area [7-11] and in other science branches in future [12-15].

\section{References}

[1] J. Von Neumann, The theory of self-reproducing automata, Ed. A.W. Burks, Univ. of Illinois Press, Urbana 1966.

[2] S. Wolfram, Rev. Mod. Phys. 55, 601 (1983).

[3] P.P. Choudhury, B.K. Nayak, S. Sahoo, S.P. Rath, arXiv:0804.2346 (2005).

[4] K. Dihidar, P.P. Choudhury, Inf. Sci. 165, 91 (2004).

[5] H. Akın, Appl. Math. Computation 170, 339 (2005).

[6] H. Akın, I. Siap, Infor. Proces. Letters 103, 24 (2007).

[7] J. Gravner, G. Gliner, M. Pelfrey, Phys. D 240, 1460 (2011).

[8] U. Sahin, F. Sahin, S. Uguz, in: 10th Int. Conf. IEEE, 2013, p. 228.

[9] U. Sahin, S. Uguz, H. Akin, Int. J. Bifurcation Chaos 25, 1550011 (2015).

[10] U. Sahin, S. Uguz, H. Akin, I. Siap, Appl. Math. Model. 39, 2003 (2015).

[11] U. Sahin, S. Uguz, F. Sahin, Comput. Electr. Eng. 40, 59 (2014).

[12] I. Siap, H. Akın, S. Uguz, Comput. Math. Applic. 62, 4161 (2011).

[13] I. Siap, H. Akın, F. Sah, J. Frank. Inst. 348, 1258 (2011).

[14] I. Siap, H. Akın, F. Sah, Infor. Sciences 180, 3562 (2010).

[15] S. Uguz, H. Akın, I. Siap, Intern. J. Bifur. Chaos 23, 1350101 (2013).

[16] S. Uguz, U. Sahin, H. Akın, I. Siap, Intern. J. Bifur. Chaos 24, 1430002 (2014).

[17] S. Uguz, U. Sahin, H. Akın, I. Siap, Acta Phys. Pol. A 125, 435 (2014). 
[18] S. Uguz, U. Sahin, F. Sahin, Comput. Electr. Eng. 43, $180(2015)$

[19] S. Uguz, H. Akin, I. Siap, U. Sahin, Appl. Math. Model. 40, 8017 (2016).

[20] P. Chattopdhyay, P.P. Choudhury, K. Dihidar, Comput. Math. App. 38, 207 (1999).
[21] H. Akın, I. Siap, S. Uguz, AIP Conf. Proceed. 1309, 16 (2010).

[22] P.P. Choudhury, S. Sahoo, S.S. Hassan, S. Basu, D. Ghosh, D. Kar, Ab. Ghosh, Av. Ghosh, A.K. Ghosh, Int. J. Comp. Cogn. 8, 50 (2010).

[23] H.H. Chou, J.A. Reggia, Phys. D 110, 252 (1997). 